\title{
Recurrencia y características clínico patológicas en los grupos de riesgo establecidos por Oncotype Dx, original y TAILORx, en cáncer de mama temprano
}

\author{
Recurrence and clinical-pathologic characteristics \\ in the risk groups stated by Oncotype Dx, original \\ and TAILORX, in early stage breast cancer
}

\author{
Geovani Amador García, ${ }^{*}$ Raúl Alejandro Andrade Moreno, ${ }^{*}$ José Fabián Martínez Herrera, ${ }^{*, \neq}$ \\ Raquel Gerson Cwilich, ${ }^{*} \S$ Juan Alberto Serrano Olvera*
}

Citar como: Amador GG, Andrade MRA, Martínez HJF, Gerson CR, Serrano OJA. Recurrencia y características clínico patológicas en los grupos de riesgo establecidos por Oncotype Dx, original y TAILORx, en cáncer de mama temprano. An Med ABC. 2021; 66 (4): 263-272. https://dx.doi.org/10.35366/102926

\section{RESUMEN}

Introducción: Oncotype $\mathrm{Dx}^{\circledR}$ predice el riesgo de recurrencia y asiste la prescripción de quimioterapia adyuvante en cáncer de mama temprano con receptores hormonales positivos, HER2 negativo y ganglios negativos o hasta tres positivos. Objetivo: Determinar la frecuencia de recurrencia y las características clínico-patológicas en los grupos de riesgo establecidos por Oncotype $\mathrm{Dx}^{\circledR}$, según la puntuación de recurrencia tradicional y TAILORx. Material y métodos: Se incluyeron mujeres > 18 años con cáncer de mama temprano (estadio clínico I-IIB), $\mathrm{RH}+/ \mathrm{HER} 2$ neg y ganglios negativos o 1-3 positivos. Se analizaron las características clínico-patológicas, recurrencia y supervivencia libre de recurrencia en los grupos con riesgo bajo, intermedio y alto. Resultados: Clasificación original: riesgo bajo 72 casos $(52.94 \%)$, riesgo intermedio $49(36.03 \%)$ y riesgo alto $15(11.03 \%)$. Clasificación TAILORx: 28 pacientes $(20.59 \%)$, $86(63.24 \%)$ y $22(16.18 \%)$ tuvieron riesgo bajo, intermedio y alto. Se observó recurrencia en $13 / 88$ casos (14.8\%); la supervivencia libre de recurrencia fue $91.3,84.8$ y $55.6 \%$ para

\section{ABSTRACT}

Introduction: Oncotype $D x^{\circledR}$ predicts the risk of recurrence and supports the prescription of adjuvant chemotherapy in early breast cancer with hormone receptor positive, HER2 negative, and node negative or up to three positive. Objective: To determine the frequency of recurrence and the clinicopathological characteristics in the risk groups established by Oncotype D $x^{\circledR}$, according to the traditional recurrence score and TAILORx. Material and methods: Women > 18 years of age with early breast cancer (stage I-IIB), Hormone receptor +1 HER2 neg, and negative or 1-3 positive nodes were included. Clinicopathological characteristics, recurrence, and recurrencefree survival were analyzed in the low, intermediate and high risk groups. Results: Original classification: low risk 72 cases (52.94\%), intermediate risk 49 (36.03\%) and high risk 15 (11.03\%). TAILORx classification: 28 patients (20.59\%), $86(63.24 \%)$ and $22(16.18 \%)$ had low risk, intermediate and high risk. Recurrence was observed in 13/88 cases (14.8\%); the recurrence free survival was $91.3,84.8$ and $55.6 \%$ for the
* Oncología Médica, Centro Médico ABC; Ciudad de México.

‡ Latin American Network for Cancer Research (LAN-CANCER), Lima, Perú.

$\S$ Directora del Centro de Cáncer, Centro Médico ABC; Ciudad de México.
Correspondencia: Dr. Juan Alberto Serrano Olvera

E-mail: serranoolvera@gmail.com

Recibido: 26/05/2021. Aceptado: 12/11/2021. 
los grupos de riesgo bajo, intermedio y alto en las categorías originales y $100,86.2$ y $66.7 \%$ en esos grupos según TAILORx. Conclusiones: La puntuación TAILORx distingue con mayor precisión al grupo de alto riesgo de recurrencia (16.8\%), en cáncer de mama temprano, receptores hormonales positivos, HER2 negativo que requiere quimioterapia adyuvante, el cual se caracteriza por menor expresión de receptores de estrógenos y supervivencia libre de recurrencia.

Palabras clave: Cáncer de mama, Oncotype Dx, riesgo de recurrencia, tratamiento adyuvante.

Nivel de evidencia: II

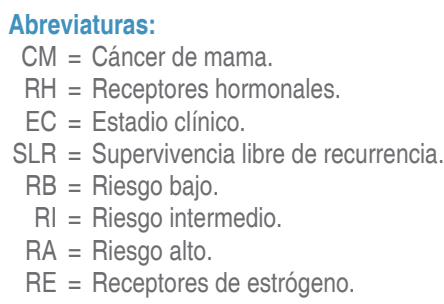

\section{INTRODUCCIÓN}

El cáncer de mama $(\mathrm{CM})$ es la primera causa de cáncer en las mujeres y en la población general, de acuerdo con GLOBOCAN 2.2 millones de nuevos casos fueron esperados en $2020^{1}$ (11.7\%), lo que significa que aproximadamente uno de cada 10 casos nuevos con cáncer es un CM; además, esta enfermedad es la primera causa de muerte oncológica en mujeres, la cual representa $15 \%$ de la totalidad de las defunciones. ${ }^{2,3}$

Se denomina etapa temprana a la presencia de tumores de mama en estadio clínico IA hasta IIB, incluye tumores T1 a T3 $(<20 \mathrm{~mm}$ hasta $>50 \mathrm{~mm}$ ), ganglios axilares N0-N1 (sin ganglios afectados o metástasis móviles a ganglios axilares ipsilaterales en los niveles I y II); en el caso de la estadificación patológica $\mathrm{pN} 0-\mathrm{pN} 1$ (sin ganglios linfáticos regionales o micrometástasis o metástasis en 1-3 ganglios linfáticos axilares y/o macrometástasis por ganglio centinela en los ganglios mamarios internos cínicamente negativos) y sin evidencia de enfermedad a distancia (M0). ${ }^{4}$

En los tumores con receptores hormonales $(\mathrm{RH})$ positivos y HER2 negativo, el tratamiento adyuvante es la terapia hormonal con antiestrogénicos durante cinco a 10 años, basada en tamoxifeno (en mujeres pre- y postmenopáusicas) o inhibidores de la aromatasa (en mujeres postmenopáusicas), lo cual reduce el riesgo de recurrencia a 10 años en $50 \%$ (RR 0.53 en low, intermediate and high risk original groups and 100 , 86.2 and $66.7 \%$ in those groups according to TAILORx's score. Conclusions: The TAILORx score is more accurate in distinguishing the group with a high risk of recurrence $(16.8 \%)$, in early breast cancer, positive hormone receptors, negative HER2 requiring adjuvant chemotherapy, which is characterized by lower estrogen receptor expression and recurrence-free survival.

Keywords: Breast cancer, Oncotype Dx, risk of recurrence, adjuvant therapy.

Level of evidence: II

los primeros cuatro años y RR 0.68 durante los años 5-9) mientras la mortalidad por CM disminuye casi un tercio durante los primeros 15 años con la terapia adyuvante (RR 0.71).5,6

La adición de terapia sistémica basada en antraciclinas y/o taxanos a la terapia hormonal ha demostrado reducir el riesgo de recurrencia en $30 \%$ en pacientes con CM temprano, con traducción en un beneficio absoluto de $5 \%$; sin embargo, $85 \%$ de las pacientes se encuentran libres de recurrencia a 10 años sólo con terapia endocrina adyuvante, entonces un porcentaje elevado de esta población podría estar sobretratada innecesariamente, agregándose toxicidad aguda y crónica en órganos vitales (cardiológica, hematológica, neurológica, gastrointestinal, entre otras); por lo anterior, es esencial distinguir aquellas mujeres que se pueden beneficiar o no de la quimioterapia adyuvante. ${ }^{7,8}$

Para reducir el sobretratamiento, es necesario determinar el estado de varios factores de alto riesgo de recurrencia, entre ellos los anatómicos (tamaño tumoral, estado ganglionar) y los biológicos (histología, tasa de proliferación, invasión linfovascular y perineural, receptores hormonales, HER2). ${ }^{9,10} \mathrm{En}$ los últimos 15 años, se han desarrollado pruebas genéticas que muestran su precisión para predecir la recurrencia del $\mathrm{CM}$, entre ellas se encuentran la prueba de 21 genes (Oncotype $\mathrm{Dx}^{\circledR}$ ), microarreglos de 70 genes (MamaPrint), 50 genes (PAM 50) o 12 genes (EndoPredict) y el Breast Cancer Index (BCI), entre otras. ${ }^{11-16}$

Oncotype $\mathrm{Dx}^{\circledR}$ es una prueba multigénica desarrollada y validada para el cálculo del riesgo de recurrencia a través del análisis de 21 genes mediante reacción en cadena de la polimerasa en tiempo real (RT-PCR) en tejido tumoral. ${ }^{17} \mathrm{~A}$ partir de 250 genes candidatos publicados en bases genómicas, obtenidos de 447 pacientes participantes en tres ensayos clínicos diferentes, se seleccionaron 21 genes asociados 
con proliferación celular (Ki67, STK 15, Survivin, CCNB1 y MYBL2), invasión (MMP11 y CTSL2), expresión de receptores de estrógenos (ER, PGR, BCL2, SCUBE2) y de la vía de HER2 (GRB7 y HER2), así como GSTM1 (glutatión transferasa), CD68 y BAG1, los cuales se compararon con cinco genes de referencia (ACTB, GAPDH, RPLPO, GUS,TFRC); posteriormente, se calculó un algoritmo que arrojó una puntuación de recurrencia (SR) que oscila entre 0 y 100 puntos, derivado de la expresión de estos genes. ${ }^{17}$

Con base en el SR, las pacientes se clasifican en riesgo bajo ( $\mathrm{RB}<18$ puntos) de recurrencia, intermedio (RI $>18$ y $<31$ puntos) y alto (RA $>31$ puntos). En los estudios de validación, la supervivencia libre de recurrencia (SLR) a 10 años fue de $93.2 \%$ en $\mathrm{RB}, 85.7 \%$ con RI y $69.5 \%$ con RA. El beneficio de la quimioterapia adyuvante fue mayor en el grupo con RA, mientras que ese beneficio no se observó en el grupo RB. El papel de la quimioterapia adyuvante no fue claro en el grupo RI. ${ }^{18,19}$

El estudio TAILORx (The Trial Assigning Individualized Options for Treatment) tuvo como objetivo primario demostrar la no inferioridad de la terapia endocrina comparada con la quimioendocrino terapia adyuvante en el grupo con riesgo intermedio, en ese trabajo se consideró un cambio en los rangos de los grupos de riesgo para minimizar el potencial de subtratamiento en los grupos de riesgo intermedio y alto. $\mathrm{Al}$ considerar que el riesgo de recurrencia es menor a $5 \%$ con tamoxifeno sólo en el grupo RB y que el beneficio de la quimioterapia adyuvante comienza a observarse a partir de los 11 puntos, los autores del estudio delimitaron los grupos RB (10 puntos o menos), RI (11-25 puntos) y RA ( $>26$ puntos). ${ }^{20}$ La SLR a nueve años del grupo RB y terapia endocrina fue $84.0 \%$, RI $83.3 \%$ con terapia endocrina y $84.3 \%$ con quimioterapia (HR $1.08, \mathrm{p}=0.26$ ) y RA $75.7 \%$, cumpliéndose el objetivo de no inferioridad de la terapia endocrina en comparación a quimioterapia en el grupo RI; sin embargo, se evidenció cierto beneficio en casos con edad menor a 50 años y SR 16 a 25 puntos. $^{20}$

El objetivo de este trabajo fue determinar las tasas de recurrencia y las características clínico patológicas en los grupos de riesgo establecidos con Oncotype $\mathrm{Dx}^{\circledR}$ (descripción original y TAILORx) en mujeres con CM temprano, RH positivos, HER2 negativo, tratadas en un centro médico privado.

\section{MATERIAL Y MÉTODOS}

Estudio retroprolectivo, observacional del primero de enero 2008 al 31 de diciembre 2018. Se incluye- ron mujeres con $\mathrm{CM}$ confirmado histopatológicamente, etapa temprana (EC 1I-IIB), mayores de 18 años, con RE y/o RP positivos (1-100\% determinado por inmunohistoquímica de acuerdo al Colegio Americano de Patología) y HER2 negativo por inmunohistoquímica $(0-1+)$ o relación HER2/CEP17 $<2.0$ confirmado por hibridación in situ en casos con resultado $2+$; ganglios axilares negativos o hasta tres positivos a metástasis, a quienes se solicitó la prueba genética de 21 genes (Oncotype $\mathrm{Dx}{ }^{\circledR}$ ). Se excluyeron casos con subtipo triple negativo, tumores $>5 \mathrm{~cm}$, carcinoma in situ, estadio IIA y IIB bajo quimioterapia neoadyuvante.

Para cada caso se registraron las siguientes variables: edad, tamaño tumoral, estadio clínico al diagnóstico, lateralidad, histología, grado de diferenciación, número de ganglios afectados, tipo de cirugía realizada, expresión de RE, RP, HER2 y Ki-67, presencia o ausencia de invasión linfovascular o perineural, puntuación de recurrencia. Los grupos de riesgo de recurrencia se definieron de acuerdo a la puntuación de recurrencia y a los puntos de corte originales de OncotypeDx ${ }^{\circledR}$ : RB $<18$ puntos, RI 19-30 y RA > 31; categorías con TAILORx: $R B \leq 10$ puntos, RI 11-25 puntos y RA > 26 puntos. La enfermedad recurrente se definió como la evidencia radiológica, clínica, bioquímica o histopatológica de actividad neoplásica a nivel local, regional o a distancia durante el seguimiento de las pacientes.

Para el cálculo de la muestra, se aplicó el método de diferencia de proporciones, esperando una diferencia teórica de $23 \%$ en las tasas de recurrencia entre los grupos de bajo y alto riesgo de recurrencia, con un error alfa de $5 \%$ a dos colas y un poder estadístico de $80 \%$, el tamaño de la muestra calculado fue 100 pacientes. Se realizó estadística descriptiva con medidas de tendencia central y de dispersión para las variables categóricas. La estadística inferencial incluyó la prueba de ANOVA de un factor para muestras independientes de tipo cuantitativo para la comparación entre grupos. Las variables categóricas fueron analizadas con prueba de $\chi^{2}$ o prueba de Fisher con menos de cinco casos. Las variables ordinales se compararon por medio de la prueba de KruskalWallis. El análisis multivariado incluyó la construcción de modelos utilizando variables con significancia estadística $(\mathrm{p}<0.05)$ para la determinación de causalidad. Se realizó análisis de SLR de acuerdo a las dos clasificaciones por el método de Kaplan-Meier. El análisis estadístico se realizó con el paquete bioestadístico SPSS v 25.0 IBM. El protocolo de este estudio fue aprobado por el Comité de Ética e Investigación. 


\section{RESULTADOS}

Se identificaron 136 casos con CM temprano y determinación del riesgo de recurrencia mediante Oncotype Dx. La edad promedio al diagnóstico fue 55.04 años, rango 30-83; mientras que la variedad histológica más frecuente fue el carcinoma ductal (86.0\%). La cirugía realizada con mayor frecuencia fue la mastectomía radical modificada en 60 casos (45.5\%) seguido de la cirugía conservadora de mama en otros $52(39.4 \%)$. Predominaron las categorías del tumor T1c y T2, 54.4 y 19.1\%. El grupo con ganglios axilares positivos representó $18.4 \%$; además, 93 pacientes $(68.4 \%)$ tenían estadio clínico IA, cinco casos (3.7\%) IB, 36 en etapa IIA (26.5\%) y dos pacientes más $(1.5 \%)$ tuvieron etapa IIB.

Todos los casos evaluados mostraron expresión positiva de RE (media de expresión $76.02 \%$ ) pero sólo $120(88.2 \%)$ tuvieron RP positivos (media 57.8\%), 105 casos $(82.0 \%)$ arrojaron expresión positiva > $50 \%$ de $\mathrm{RE}$, mientras que $63.4 \%$ tuvo $\mathrm{RP}$ positivo $>$ $50 \%$. HER2 fue negativo en $99.3 \%$ de los casos analizados, el resultado no fue concluyente por inmunohistoquímica $(2+)$ en un caso y no se encontró registro de su análisis mediante FISH.

El grado histológico bien diferenciado se informó en 39 casos (28.7\%), moderadamente diferenciado 83 $(61.0 \%)$ y poco diferenciado $14(10.3 \%)$. La proliferación celular fue elevada ( $>14 \%$ ) en 55 casos $(40.4 \%)$ y la media de expresión fue $15.9 \%$, rango $1-85 \%$.

El SR según los criterios originales ${ }^{18}$ permitió distinguir 72 pacientes $(52.94 \%)$ en el grupo RB, 49 (36.03\%) con RI y 15 (11.03\%) en el grupo RA. La expresión positiva de $\mathrm{RE}<50 \%$ fue observada con mayor frecuencia en el grupo RA (60, 82 y $87 \%$, respectivamente, $p=0.022$ ). Un comportamiento similar se notó en cuanto a la prevalencia de expresión positiva de $\mathrm{RP}<50 \%$, RB $33.3 \%$, RI $63.4 \%$, RA $75.4 \%$, p < 0.001. También el grado histológico fue mayor (grados II y III) en aquéllas del grupo RA. Ki67 > 14\% se detectó en $80 \%$ con RA, $37.5 \%$ con RB y $32.7 \%$ con $\mathrm{RI}, \mathrm{p}=0.004$; además, la invasión vasculolinfática fue mayor en el grupo con alto riesgo (RB25\%, RI $28.6 \%$ y RA $60 \%$ ), $\mathrm{p}=0.026$ (Tabla 1 ).

De acuerdo a categoría de grupos TAILORx, ${ }^{20}$ 28 pacientes $(20.59 \%)$ se clasificaron con $\mathrm{RB}, 86$ (63.24\%) RI y 22 casos (16.18\%) en RA. De la clasificación original, 44 pacientes $(61.1 \%)$ de riesgo bajo fueron reclasificadas como riesgo intermedio y siete de riesgo intermedio (14.3\%) pasaron al $\mathrm{RA}, \mathrm{p}<$ 0.0001 en RB y $\mathrm{p}<0.0001$ en RA. La edad promedio fue menor en el grupo RA $(60.6,53.67$ y 53.1 años, respectivamente), $p=0.013$. En el grupo con $\mathrm{RA}$ se observó mayor proporción de casos con $\mathrm{RE}(\mathrm{p}=$ $0.004)$ y RP positivos $<50 \%$ ( $<<0.001)$; mayor grado histológico, $\mathrm{p}=0.008$; elevada proliferación celular, $\mathrm{p}=0.001$ e invasión vasculolinfática, $\mathrm{p}=0.046$.

En relación con las categorías originales de riesgo, la quimioterapia adyuvante se prescribió a 2/72 casos del grupo RB (2.8\%), 5/49 (10.2\%) con RI y a $5 / 15(33.3 \%)$ con $R A, p=0.001$. Por otra parte, con los criterios TAILORx, se registró prescripción de quimioterapia adyuvante en $1 / 28(3.6 \%)$ con $\mathrm{RB}$, $4 / 86(4.7 \%)$ con $\mathrm{RI}$ y en $7 / 22$ pacientes $(31.8 \%)$ con $\mathrm{RA}, \mathrm{p}<0.0001$. Los esquemas de quimioterapia empleados fueron diversos, siendo los más frecuentes docetaxel/ciclofosfamida en cinco casos (41.7\%), doxorrubicina/ciclofosfamida seguido de paclitaxel en cuatro (33.3\%), un caso (8.3\%) para cada uno de los siguientes: CMF, paclitaxel monodroga o tomografía axial computada (TAC). La terapia endocrina más empleada fue tamoxifeno, en las mujeres premenopaúsicas así como un inhibidor de la aromatasa entre la postmenopaúsicas.

La mediana del tiempo de seguimiento fue 11.6 años, (rango $9.1 \mathrm{~m}-12.4$ años), durante este periodo 48 casos $(35.3 \%)$ se perdieron del seguimiento; de las restantes 88 mujeres, se detectó recurrencia en 13 (14.8\%) de ellas. La mediana de SLR fue 131.59 meses, (rango 9.1-149.13 meses). En los grupos de riesgo descritos por Paik ${ }^{18}$ la recurrencia se documentó en cuatro casos $(8.7 \%)$ con $\mathrm{RB}$, cinco $(15.2 \%)$ con $\mathrm{RI}$ y cuatro $(44.4 \%)$ con $\mathrm{RA}, \mathrm{p}=0.022$, mientras con TAILORx no se documentó recurrencia con $\mathrm{RB}$, pero RI y RA se registró en ocho (13.8\%) y cinco (33.3\%) respectivamente, $\mathrm{p}=0.034$ (Tabla 2 ). En relación al número de sitios de recurrencia y localización de la recurrencia, no se observó diferencia (Tabla 3).

En los grupos de riesgo originales, la tasa de SLR a 11.6 años fue RB $91.3 \%$, RI $84.8 \%$ y RA $55.6 \%$ (Log Rank 0.017); la mediana de SLR fue 138.1, 126.1 y 96.4 meses, respectivamente (Figura 1). Por otra parte, en los grupos de riesgo TAILORx, la tasa de SLR fue RB $100 \%$, RI 86.2 y $66.7 \%$ con RA (Log Rank 0.025), la mediana de SLR fue 132.2 y 100.6 meses RI y RA (Figura 2). El análisis multivariado de Cox evidenció que la expresión de $\mathrm{RE}$ $<50 \%$ (HR 5.191, IC05\% 1.345-19.886), RP < 50\% (HR1.508, IC95\% 0.426-5.436) e invasión linfovascular (HR 1.191, IC95\% 0.702-0.124) fueron factores asociados al desarrollo de recurrencia sistémica; sin embargo, la única variable que retuvo diferencia estadística fue la expresión de $\mathrm{RE}<50 \%, \mathrm{p}=0.016$ (Tabla 4). 
An Med ABC. 2021; 66 (4): 263-272

Tabla 1: Características generales.

\begin{tabular}{|c|c|c|c|c|c|c|c|c|c|}
\hline & \multirow{2}{*}{ Total } & \multicolumn{3}{|c|}{ Original } & \multirow{2}{*}{$p$} & \multicolumn{3}{|c|}{ TAILORx } & \multirow{2}{*}{$p$} \\
\hline & & Bajo & Intermedio & Alto & & Bajo & Intermedio & Alto & \\
\hline Pacientes & 136 & $72(52.9)$ & $49(36.03)$ & $15(11.03)$ & & $28(20.59)$ & 86 (63.24) & $22(16.18)$ & \\
\hline Edad (media) & 55.04 & 55.52 & 55.26 & 51.93 & 0.854 & 60.68 & 53.67 & 53.18 & 0.013 \\
\hline Ductal invasor & $117(86.0)$ & 64 (88.9) & $38(77.6)$ & $15(100)$ & & $24(85.7)$ & $73(84.9)$ & $20(90.9)$ & \\
\hline Lobulillar invasor & $11(8.1)$ & $4(5.6)$ & 7 (14.3) & $0(0)$ & 0.115 & 1 (3.6) & $8(9.3)$ & $2(9.1)$ & 0.729 \\
\hline Mucinoso & $5(3.7)$ & $2(2.8)$ & $3(6.1)$ & $0(0)$ & & $1(3.6)$ & $4(4.7)$ & $0(0.0)$ & \\
\hline Mixto & $3(2.2)$ & $2(2.8)$ & $1(2.0)$ & $0(0)$ & & $2(7.1)$ & $1(1.2)$ & $0(0.0)$ & \\
\hline \multicolumn{10}{|l|}{ Cirugía } \\
\hline MRM & $60(45.5)$ & $35(51.5)$ & 19 (38.8) & $6(40.0)$ & & $12(46.2)$ & 38 (45.2) & $10(45.5)$ & \\
\hline \multicolumn{10}{|l|}{ Tamaño tumoral } \\
\hline T1mi & $1(0.7)$ & $1(1.4)$ & $0(0.0)$ & $0(0.0)$ & & $1(3.6)$ & $0(0.0)$ & $0(0.0)$ & \\
\hline T1a & $4(2.9)$ & $3(4.2)$ & $1(2.0)$ & $0(0.0)$ & 0.858 & $0(0.0)$ & $4(4.7)$ & $0(0.0)$ & 0.815 \\
\hline $\mathrm{T} 1 \mathrm{~b}$ & $31(22.8)$ & 16 (22.2) & $10(20.4)$ & 5 (33.3) & & $6(21.4)$ & $19(22.1)$ & $6(27.3)$ & \\
\hline $\mathrm{T} 1 \mathrm{C}$ & $74(54.4)$ & 39 (54.2) & $29(59.2)$ & $6(40.0)$ & & $16(57.1)$ & $48(55.8)$ & $10(45.5)$ & \\
\hline T2 & $26(19.1)$ & 13 (18.1) & $9(18.4)$ & $4(26.7)$ & & $5(17.9)$ & $15(17.4)$ & $6(27.3)$ & \\
\hline \multicolumn{10}{|l|}{ Ganglios } \\
\hline pNO & $111(81.6)$ & $64(88.9)$ & 35 (71.4) & $12(80.0)$ & 0.052 & 25 (89.3) & 70 (81.4) & $16(72.7)$ & 0.443 \\
\hline pN1 & $25(18.4)$ & $8(11.1)$ & 14 (28.6) & $3(20.0)$ & & $3(10.7)$ & $16(18.6)$ & $6(27.3)$ & \\
\hline \multicolumn{10}{|l|}{ Etapa clínica } \\
\hline IA & $93(68.4)$ & $56(77.8)$ & $28(57.1)$ & $9(60.0)$ & 0.139 & $21(75.0)$ & $60(69.8)$ & $12(54.5)$ & 0.202 \\
\hline Positivo & $120(88.2)$ & 70 (97.2) & $42(85.7)$ & 8 (53.3) & - & 27 (96.4) & $81(94.2)$ & $12(54.5)$ & - \\
\hline Negativo & $16(11.8)$ & $2(2.8)$ & 7 (14.3) & $7(46.7)$ & & $1(3.6)$ & $5(5.8)$ & $10(45.5)$ & \\
\hline RE \% media & 76.02 & 75.94 & 75.79 & 76.02 & 0.483 & 76.85 & 75.94 & 76.02 & 0.650 \\
\hline RP \% media & 57.8 & 59.3 & 58.56 & 59.71 & 0.339 & 59.0 & 59.34 & 59.71 & 0.371 \\
\hline $\mathrm{RE}>50 \%$ & $105(82.0)$ & $60(87.0)$ & $36(81.8)$ & $9(60.0)$ & 0.022 & $27(100.0)$ & 65 (80.2) & $13(65.0)$ & 0.004 \\
\hline $\mathrm{RP}>50 \%$ & $83(63.4)$ & $52(75.4)$ & $26(55.3)$ & 5 (33.3) & $<0.001$ & $24(88.9)$ & $51(61.4)$ & 8 (38.1) & $<0.001$ \\
\hline Her2 (-) & 135 (99.3) & $72(100.0)$ & $49(100.0)$ & 14 (93.3) & & $28(100.0)$ & $86(100.0)$ & $21(95.5)$ & \\
\hline \multicolumn{10}{|l|}{ Grado Dif. } \\
\hline G1 & $39(28.7)$ & 27 (37.5) & $11(22.4)$ & $1(6.7)$ & 0.010 & $9(32.1)$ & $29(33.7)$ & $1(4.5)$ & 0.008 \\
\hline $\mathrm{G} 2$ & $83(61.0)$ & $41(56.9)$ & 31 (63.3) & $11(73.3)$ & & $17(60.7)$ & $50(58.1)$ & $16(72.7)$ & \\
\hline G3 & $14(10.3)$ & $4(5.6)$ & 7 (14.3) & $3(20.0)$ & & $2(7.1)$ & $7(8.1)$ & $5(22.7)$ & \\
\hline Ki67 media & 15.90 & 12.86 & 15.04 & 33.35 & 0.079 & 13.34 & 12.56 & 32.24 & 0.088 \\
\hline Ki67 > 14\% & $55(40.4)$ & 27 (37.5) & 16 (32.7) & $12(80.0)$ & 0.004 & 10 (35.7) & 28 (32.6) & 17 (77.3) & 0.001 \\
\hline ILV+ & $41(30.1)$ & $18(25.0)$ & 14 (28.6) & $9(60.0)$ & 0.026 & $5(17.9)$ & $25(29.1)$ & $11(50.0)$ & 0.046 \\
\hline IPN+ & 29 (21.3) & $15(20.8)$ & $9(18.4)$ & 5 (33.3) & 0.460 & $22(78.6)$ & $69(80.2)$ & $16(72.7)$ & 0.745 \\
\hline
\end{tabular}

MRM = mastecomía radica modificada, MS = mastectomía simple, MCCAP = mastectomía conservadora de complejo areola pezón, $C C$ = cirugía conservadora, $\mathrm{RE}=$ receptores de estrógenos, $\mathrm{RP}=$ receptores de progesterona, ILV = invasión linfovascular, IPN = invasión perineural. 


\section{DISCUSIÓN}

En México, se han publicado dos trabajos sobre este tema en particular. Gerson y colaboradores ${ }^{21}$ reportaron similar prevalencia de los grupos de riesgo al utilizar los puntos de corte originales $(63.9,22.2$ y $13.9 \%$ ); el otro reporte mexicano usó los puntos de corte descritos por Paik ${ }^{18}$ y encontró menor proporción de casos con RB (48\%), mayor proporción de casos con RI (31\%) y RA (21\%) entre 96 casos de una Institución Pública; ${ }^{22}$ posiblemente, esas diferencias estén asociadas al mayor tamaño tumoral (38\% con tumores de $2-4 \mathrm{~cm}$ y $7 \%$ con tamaño $>4 \mathrm{~cm}$ ) y mayor proporción de casos con ganglios axilares positivos (34\% con uno a tres positivos).

Aquí, las características clínico-patológicas asociadas al grupo RA fueron, en ambos sistemas de categorización, la expresión < 50\% de RE y RP, elevado Ki67 y presencia de invasión linfovascular, aunque sólo $\mathrm{RE}<50 \%$ retuvo diferencia estadística en el análisis multivariado. Paik y colaboradores ${ }^{18}$ informaron mayor tasa de recurrencia a distancia en pacientes menores de 50 años (21.1 vs $12.3 \%$ ), tumor mayor a $2 \mathrm{~cm}(17.5$ vs $13.3 \%)$ y tumores poco diferenciados. Por otra parte, Sparano y su equipo ${ }^{20}$

Tabla 2: Riesgo de recurrencia por grupo.

\begin{tabular}{lccc} 
& \multicolumn{2}{c}{ Grupo de riesgo original } & \\
\cline { 2 - 3 } $\begin{array}{l}\text { Presencia de } \\
\text { recurrencia }\end{array}$ & $\begin{array}{c}\text { Bajo } \\
\mathrm{n}(\%)\end{array}$ & $\begin{array}{c}\text { Intermedio } \\
\mathrm{n}(\%)\end{array}$ & $\begin{array}{c}\text { Alto } \\
\mathrm{n}(\%)\end{array}$ \\
\hline Sí & $4(8.7)$ & $5(15.2)$ & $4(44.4)$ \\
No & $42(91.3)$ & $28(84.8)$ & $5(55.6)$ \\
\hline \multicolumn{4}{c}{ Grupo de riesgo TAILORx } \\
\hline Sí & $0(0.0)$ & $8(13.8)$ & $5(33.3)$ \\
No & $15(100.0)$ & $50(86.2)$ & $10(66.7)$ \\
\hline
\end{tabular}

describieron que en el grupo con la puntuación de recurrencia $>26$ puntos (grupo RA) había mayor proporción de casos con expresión negativa de $\mathrm{RE}$ (3\%), de progesterona (30\%) y grado histológico alto $(50 \%)$. Dichas características han sido consistentemente reportadas por varios autores (Gerson, Bargallo, Huang); ${ }^{21-23}$ recientemente, Sparano y su grupo ${ }^{24}$ señalaron que la estratificación clínica basada en el tamaño tumoral y grado histológico provee información pronóstica adicional al perfil de riesgo de 21 genes, principalmente en mujeres premenopáusicas. Por lo anterior, Zhang y colaboradores ${ }^{25}$ desarrollaron un modelo predictor del riesgo de recurrencia, basado en cuatro variables: Ki67 > 27.5\%, expresión de $\mathrm{RP}<55 \%$ o $>$ a $55 \%$ pero con riesgo clínico elevado (tamaño tumoral $>3 \mathrm{~cm}$ y/o alto grado histológico), el desempeño del modelo fue contrastado con los criterios de originales de Oncotype Dx y TAILORx; en la fase de validación del modelo se estimó sensibilidad 50\%, especificidad $95.8 \%$, valor predictivo positivo $72 \%$ y predictivo negativo $89.9 \%$.

En este reporte, la quimioterapia adyuvante fue prescrita en 12/136 casos (8.8\%), según la categoría original y TAILORx, RB (2.8 vs $3.6 \%)$, RI (10.2 vs $4.7 \%)$ y RA (33.3 vs $31.8 \%)$. La relevancia de la categorización de los grupos de riesgo, la confiabilidad de su papel pronóstico y mejor comprensión del uso de Oncotype Dx han permitido, a través de los años, reducir la prescripción de quimioterapia en los grupos con bajo o intermedio riesgo de recurrencia. En el primer reporte nacional, se informó que $25 \%$ de los casos analizados fue tratado con quimioterapia adyuvante, aunque $58 \%$ fue considerado para dicha modalidad terapéutica previo al resultado de Oncotype Dx. ${ }^{21} \mathrm{El}$ otro estudio nacional indicó que la recomendación de quimioterapia disminuyó de 48 a $34 \%$ en los 96 casos analizados y de 59 a $32 \%$ en casos con ganglios axilares positivos, debido al resultado del estudio genómico. ${ }^{22}$

Tabla 3: Número y sitios de recurrencia por grupo de riesgo.

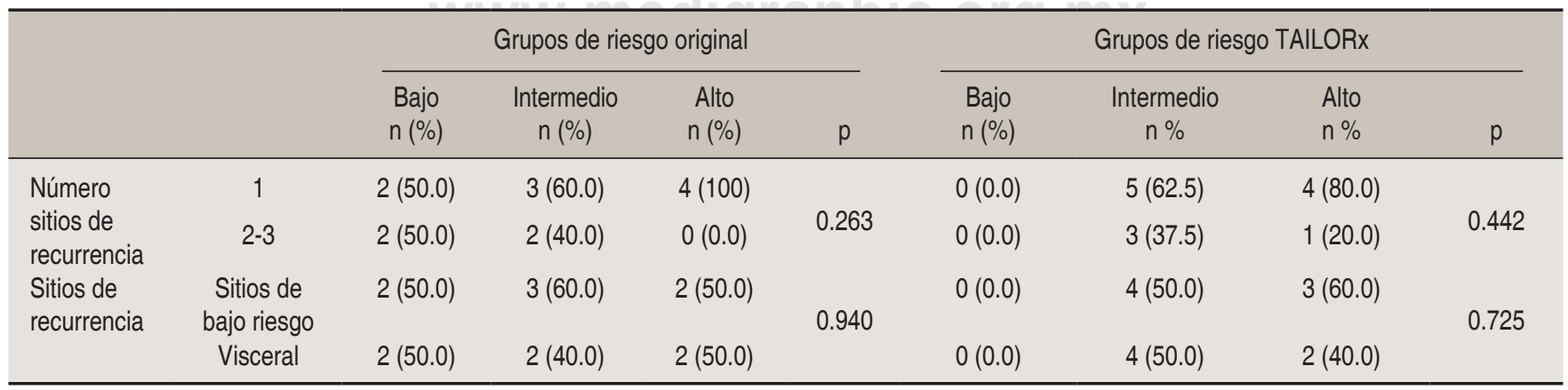




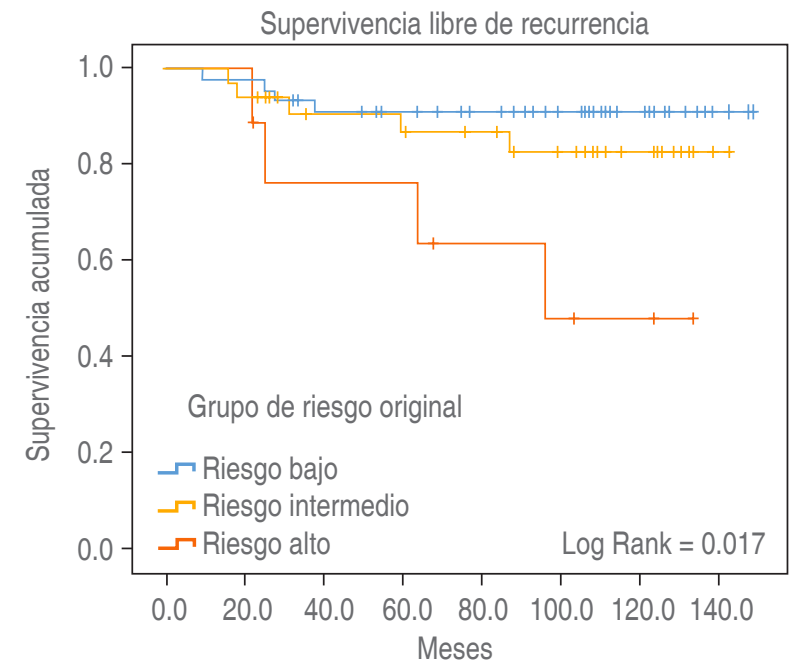

\begin{tabular}{|c|c|c|}
\hline Grupo de riesgo & Media SLR $(\mathrm{m})$ & IC $95 \%$ \\
\hline Bajo & 138.1 & $127.8-148.4$ \\
\hline Intermedio & 126.1 & $112.4-139.9$ \\
\hline Alto & 92.8 & $61.6-124.1$ \\
\hline
\end{tabular}

Figura 1: Supervivencia libre de recurrencia en los grupos de riesgo original.

En nuestro trabajo, los esquemas de quimioterapia basados en taxanos sin antraciclinas (TC) o compuestos por antraciclinas seguidos de un taxano (ACP) fueron los más frecuentes. Estas combinaciones de fármacos han sido empleadas desde hace varios años; en la actualidad, no hay consenso sobre cuál esquema sea el mejor en la adyuvancia del CM temprano. En el ensayo TAILORx la combinación de docetaxel y ciclofosfamida, así como los esquemas que contienen antraciclinas fueron los que se indicaron con mayor frecuencia. ${ }^{20} \mathrm{Un}$ estudio retrospectivo en $\mathrm{CM}$, etapa I-III, con RH positivos y HER2 negativo a quienes se realizó Oncotype Dx analizó la prescripción de quimioterapia adyuvante basada en antraciclinas o libre de ellas en función de los grupos de riesgo, edad, etapa clínica, grado tumoral y estado de los receptores de progesterona; entre 6,741 casos con puntuación de riesgo, los autores informaron que el uso de antraciclinas aumentó conforme el riesgo de recurrencia era mayor, RB $1.6 \%$, RI $8.2 \%$ y RA $33.6 \%$; también, este tipo de agentes antitumorales fue utilizado con mayor frecuencia en menores de 65 años (40 vs 6.4\%) y ganglios axilares positivos (26.2 vs 6.6\%). Los autores señalaron que la prescripción de las antraciclinas fue más frecuente aún en pacientes con RB o RI con ganglios positivos; sin embargo, el estado ganglionar axilar no parece haber sido un factor de selección del tratamiento en el grupo con RA. ${ }^{26}$
La esencia del estudio publicado por Paik ${ }^{18}$ fue validar, de manera prospectiva, la capacidad de la prueba de 21 genes para predecir el riesgo de recurrencia en casos con ganglios negativos y RE positivos tratados con tamoxifeno adyuvante, sus resultados rápidamente derivaron en considerar que el grupo RA debía recibir quimio y endocrinoterapia y, ante la carencia de información, la quimioterapia seguida de terapia hormonal era indicada en casos con RI y ganglios positivos. ${ }^{27}$ TAILORx ${ }^{20}$ fue un ensayo controlado, prospectivo, el cual analizó 10,253 mujeres de 18 a 75 años, con CM, RH positivos, HER2 negativo y ganglios axilares negativos, el cual fue dirigido para evaluar la no inferioridad de la terapia endocrina frente a la quimioterapia adyuvante en casos con RI (puntuación 11-25). Los autores concluyeron que la terapia hormonal adyuvante tiene una eficacia similar a la quimio y terapia endocrina en términos de la supervivencia libre de enfermedad invasiva, recurrencia a distancia, locorregional y supervivencia global, por lo anterior, la quimioterapia adyuvante ha quedado reservada sólo para los casos con RA y mujeres jóvenes.

En el presente trabajo, ni la eficacia aislada para la terapia endocrina ni la proporción de cambio en la recomendación del tipo de terapia adyuvante fueron evaluadas. Gerson y colaboradores ${ }^{21}$ reportaron que $69 \%$ del grupo RI y $100 \%$ con RA recibieron quimio-

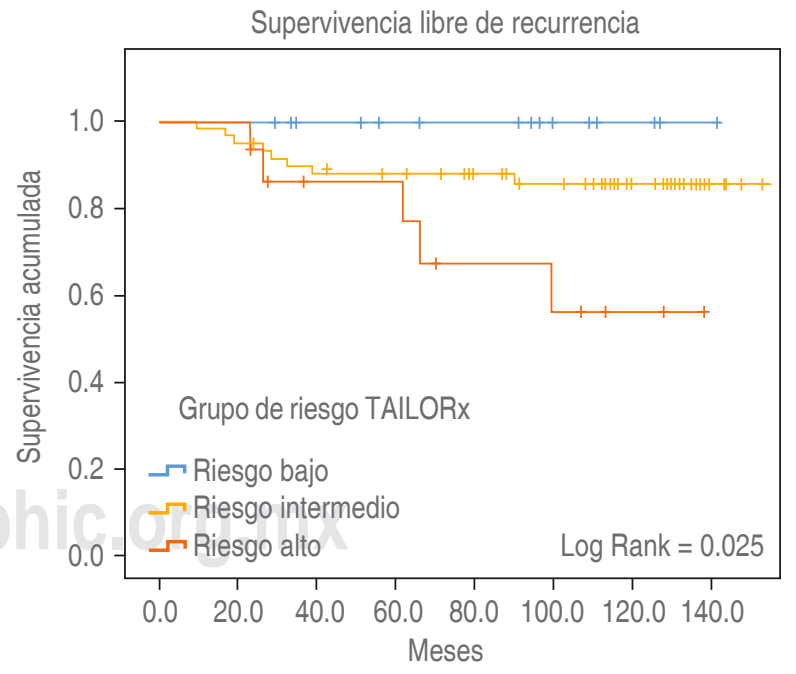

\begin{tabular}{|c|c|c|}
\hline Grupo de riesgo & Media SLR $(\mathrm{m})$ & IC $95 \%$ \\
\hline Intermedio & 132.2 & $121.3-143.2$ \\
\hline Alto & 100.6 & $77.3-124.0$ \\
\hline
\end{tabular}

Figura 2: Supervivencia libre de recurrencia en los grupos de riesgo TAILORX. 
Tabla 4: Análisis multivariado de Cox para recurrencia sistémica.

\begin{tabular}{|c|c|c|c|c|c|c|}
\hline \multicolumn{7}{|c|}{ Regresión de Cox } \\
\hline & Beta & $\begin{array}{l}\text { Grados de } \\
\text { libertad }\end{array}$ & $p$ & Hazard ratio & \multicolumn{2}{|c|}{ IC 95\% } \\
\hline $\mathrm{RE}<50 \%$ & 1.647 & 1 & 0.016 & 5.191 & 1.355 & 19.886 \\
\hline $\mathrm{RP}<50 \%$ & 0.411 & 1 & 0.524 & 1.508 & 0.426 & 5.346 \\
\hline Grado histológico & & 2 & 0.310 & & & \\
\hline 1 & -1.476 & 1 & 0.138 & 0.229 & 0.033 & 1.607 \\
\hline 2 & -0.420 & 1 & 0.597 & 0.657 & 0.138 & 3.124 \\
\hline Invasión linfovascular & 0.175 & 1 & 0.802 & 1.191 & 0.304 & 4.664 \\
\hline Invasión perineural & -0.354 & 1 & 0.689 & 0.702 & 0.124 & 3.964 \\
\hline
\end{tabular}

terapia y se informó cambio de recomendación terapéutica en $57 \%$; posiblemente, esta diferencia refleja un cambio de la cultura médica a través del aprendizaje y mejor entendimiento del uso de Oncotype Dx. Bargalló y su equipo ${ }^{22}$ señalaron que la decisión de tratamiento sistémico se modificó en $32 \%$ de los casos debido al resultado de la prueba de 21 genes, el cambio de recomendación terapéutica fue mayor en el grupo con ganglios positivos en comparación con aquéllas sin afectación ganglionar, 41 vs $27 \%$. Albain y su equipo ${ }^{28}$ en un estudio de revisión informaron que el resultado de Oncotype Dx fue capaz de modificar la prescripción de la terapia adyuvante entre 18 y $44 \%$ de los casos.

En esta población, la enfermedad recurrente se observó en 13/88 (14.8\%) casos; para los grupos RB, RI y RA de la categoría original ésta fue detectada en $8.7,15.2$ y $44.4 \%$ en comparación con $0,13.8$ y $33.3 \%$ de los casos, respectivamente según los puntos de cohorte del TAILORx. Dos o más sitios de recurrencia en el grupo RA fueron más frecuentes con los criterios TAILORx vs originales (20 vs $0 \%$ ) mientras que el involucro de órganos viscerales fue mayor en el grupo RA, (50 vs 40\%). Ninguno de los estudios nacionales, previamente publicados, determinó la frecuencia de recurrencia en los distintos grupos de riesgo (Gerson, Bargalló). ${ }^{21,22}$ Comparativamente, Paik $^{18}$ estimó la tasa de recurrencia a distancia a 10 años en $6.8,14.3$ y $30.5 \%$ en los grupos con bajo, intermedio y alto riesgo, respectivamente; estas cifras fueron similares a las observadas en este trabajo.

El ensayo TAILORx intentó determinar con mayor certeza la tasa de recurrencia en cada grupo de riesgo y la no inferioridad al tratar con terapia endo- crina o quimioterapia al grupo $\mathrm{RI} ;{ }^{20}$ en ese estudio se consideró la recurrencia como una variable compuesta donde se incluyó la recurrencia local, locorregional, contralateral, a distancia, segundas neoplasias primarias mamarias y la defunción; la frecuencia de eventos fue $11.4 \%$ en el grupo con puntuación $<10$ (RB), $13.6 \%$ con puntuación $>26$ (RA), en el grupo con puntuación 11-25 (RI) que fueron tratados sólo con terapia endocrina la prevalencia fue $12.8 \%$ y en aquéllos con la misma puntuación tratadas con quimioterapia fue $12.1 \%$; sin embargo, en relación con la recurrencia a distancia, ésta fue detectada en $28 / 1,619$ con RB, 107/3,339 con RI y terapia endocrina, 92/3,312 con RI y quimioendocrinoterapia, $80 / 1,389$ casos con RA.

En la población de nuestro centro, la tasa y mediana de SLR a 11.6 años fue menor en el grupo RA, tanto con los puntos de corte tradicionales y TAILORx. El ensayo TAILORx ${ }^{20}$ fue enfocado en determinar la no inferioridad de la terapia endocrina adyuvante en los grupos con riesgo bajo e intermedio, lo cual se tradujo en similares tasas de supervivencia libre de enfermedad invasiva, recurrencia a distancia o locorregional, así como supervivencia global a cinco y nueve años. Los resultados del ensayo corroboraron la hipótesis de trabajo, los grupos con riesgo bajo o intermedio tratados con terapia endocrina o quimioendocrinoterapia cursaron con similares expectativas en los rubros evaluados; sin embargo, el grupo con riesgo elevado tuvo peores expectativas de control locorregional y a distancia así como en supervivencia global.

Hasta el momento, el presente trabajo es el que ha analizado los grupos de riesgo de Oncotype Dx en el 
mayor número de casos con CM temprano en México; además, es el único estudio nacional que ha realizado la comparación de variables clínico patológicas y recurrencia tumoral en función de los puntos de corte propuestos originalmente por Paik y colaboradores ${ }^{18} \mathrm{y}$ aquéllos empleados en el estudio TAILORx ${ }^{20}$. No obstante, los resultados de la presente investigación deben ser interpretados con cautela, ya que existen algunas condiciones metodológicas que limitan su validez externa, entre ellas el carácter observacional y retrospectivo, el tamaño pequeño de la muestra, así como la pérdida de seguimiento del $35.3 \%$ de los casos. Además, este trabajo no fue capaz de explorar el efecto de la quimioterapia adyuvante en el grupo RI-TAILORx y tampoco pretendió evaluar la proporción de cambio en las recomendaciones del tratamiento adyuvante.

\section{CONCLUSIONES}

La puntuación de riesgo de recurrencia determinado mediante Oncotype Dx identifica tres grupos del cáncer de mama temprano, receptores hormonales positivos, HER2 negativo y ganglios axilares negativos o hasta tres positivos, los más frecuentes son el de riesgo bajo o intermedio. Los puntos de corte TAILORx detectan con mayor precisión los grupos que no requieren quimioterapia adyuvante. El grupo de alto riesgo es caracterizado por menor edad y menor expresión positiva de receptores estrogénicos. La recurrencia de la enfermedad y la supervivencia libre de recurrencia a largo plazo son mayores en los grupos con riesgo bajo o intermedio en comparación al de riesgo alto. Para este grupo de mujeres con cáncer de mama temprano es indispensable contar con esta herramienta genómica con el objetivo de seleccionar correctamente quiénes pueden prescindir o no de la quimioterapia adyuvante.

\section{REFERENCIAS}

1. Sung H, Ferlay J, Siegel RL, Laversanne M, Soerjomataram I, Jemal A et al. Global cancer statistics 2020: GLOBOCAN estimates of incidence and mortality worldwide for 36 cancers in 185 countries. CA Cancer J Clin. 2021; 71 (3): 209-249. doi: 10.3322/caac.21660.

2. American Cancer Society. Breast Cancer Facts \& Figures 20192020. Atlanta: American Cancer Society, Inc, 2019. [Consulted 26 February 2021] Available in: https:/www.cancer.org/ content/dam/cancer-org/research/cancer-facts-and-statistics/ breast-cancer-facts-and-figures/breast-cancer-facts-andfigures-2019-2020.pdf

3. Walks AG, Winer EP. Breast cancer treatment: a review. JAMA. 2019; 321 (3): 288-300.

4. Kim EJ, Park HS, Kim JY, Kim SI, Cho YU, Park BW. Assessment of the Prognostic Staging System of American
Joint Committee on Cancer 8th Edition for Breast Cancer: Comparisons with the Conventional Anatomic Staging System. J Breast Cancer. 2020; 23 (1): 59-68.

5. Davies C, Godwin J, Gray R, Clarke M, Darby S, McGale P et al. Early Breast Cancer Trialists' Collaborative Group (EBCTCG). Relevance of breast cancer hormone receptors and other factors to the efficacy of adjuvant tamoxifen: patientlevel meta-analysis of randomised trials. Lancet. 2011; 378: 771-784.

6. Clarke M, Collins R, Davies C, Godwin J, Gran R, Peto R. Tamoxifen for early breast cancer: an overview of the randomised trials. Early Breast Cancer Trialists' Collaborative Group. Lancet. 1998; 351 (9114): 1451-1467.

7. Peto R, Davies C, Godwin J, Gray R, Pan H C, Clarke M et al. Early Breast Cancer Trialists' Collaborative Group (EBCTCG). Comparisons between different polychemotherapy regimens for early breast cancer: meta-analyses of long-term outcome among 100,000 women in 123 randomised trials. Lancet. 2012; 379: 432-444.

8. Sparano JA, Paik S. Development of the 21-Gene assay and its application in clinical practice and clinical trials. J Clin Oncol 2008; 26 (5): 721-728.

9. Cianfrocca M, Goldstein LJ. Prognostic and predictive factors in early-stage breast cancer. Oncologist. 2004; 9 (6): 606-616.

10. Rakha EA, Reis-Fihlo JS, Baehner F, Dabbs DJ, Decker T, Eusebi V et al. Breast Cancer prognostic classification in the molecular era: the role of histological grade. Breast Cancer Res. 2010; 12 (4): 207.

11. Van't Veer LJ, Dai H, van de Vijver MJ, He YD, Hart AA, Mao $\mathrm{M}$ et al. Gene expression profiling predicts clinical outcome of breast cancer. Nature. 2002; 415: 530-36.

12. Cardoso F, van't Veer LJ, Bogaerts J, Slaets L, Viale G, Delaloge $\mathrm{S}$ et al. 70-Gene signature as an aid to treatment decisions in early-stage breast cancer. N Engl J Med. 2016; 375 (8): 717-729.

13. Parker JS, Mullins M, Cheang MCU, Leung S, Voduc D, Vivkery $\mathrm{T}$ et al. Supervised risk predictor of breast cancer based on intrinsic subtypes. J Clin Oncol. 2009; 27: 11601167.

14. Dowsett M, Sestak I, Lopez-Knowles E, Sidhu K, Dunbier AK, Cowens JW et al. Comparison of PAM50 risk of recurrence score with oncotype DX and IHC4 for predicting risk of distant recurrence after endocrine therapy. J Clin Oncol. 2013; 31: 2783-2790.

15. Filipits M, Rudas M, Jakesz R, Dubsky P, Fitzal F, Singer $\mathrm{CF}$ et al. A new molecular predictor of distant recurrence in ER-positive, HER2-negative breast cancer adds independent information to conventional clinical risk factors. Clin Cancer Res. 2011; 17 (18): 6012-6020.

16. Sestak I, Buus R, Cuzick J, Dubsky P, Kronenwett R, Denkert $\mathrm{C}$ et al. Comparison of the performance of 6 prognostic signatures for estrogen receptor-positive breast cancer: a secondary analysis of a randomized clinical trial. JAMA Oncol. 2018; 4 (4): 545-553.

17. Sparano J, Paik S. Development of the 21-gene assay and its application in clinical practice and clinical trials. J Clin Oncol. 2008; 26: 721-728.

18. Paik S, Shak S, Tang G, Kim C, Baker J, Cronin M et al. A multigene assay to predict recurrence of tamoxifen-treated, node-negative breast cancer. N Engl J Med. 2004; 351: 28172826.

19. Albain KS, Barlow WE, Shak S, Hortobagy GN, Livingston RB, Yeh IT et al. Prognostic and predictive value of the 21gene recurrence score assay in postmenopausal women with 
An Med ABC. 2021; 66 (4): 263-272

node-positive, oestrogen-receptor-positive breast cancer on chemotherapy: a retrospective analysis of a randomised trial. Lancet Oncol. 2010; 11 (1): 55-65.

20. Sparano JA, Gray RJ, Makower DF, Protchard KI, Albain KS, Hayes DF et al. Adjuvant chemotherapy guided by a 21-gene expression assay in breast cancer. N Engl J Med. 2018; 379 (2): 111-121.

21. Gerson R, Alban LF, Villalobos A, Serrano JA. Características clinicopatológicas, pronóstico e influencia en el tratamiento adyuvante en los grupos de riesgo de recurrencia determinados por el perfil de expresión de 21 genes, Oncotype Dx, en cáncer de mama temprano. Gac Med Mex. 2012; 148 (2): 117-124.

22. Bargallo JE, Lara F, Shaw-Dulin R, Pérez-Sánchez V, Villarreal-Garza C, Maldonado-Martínez H et al. A study of the impact of the 21-gene breast cancer assay on use of adjuvant chemotherapy in women with breast cancer in a Mexican public hospital. J Surg Oncol. 2015; 111: 203-207.

23. Huang JL, Kizy S, Marmor S, Altman A, Blaes A, Beckwith $\mathrm{H}$ et al. Tumor grade and progesterone receptorstatus predict 21-gene recurrence scorein early stage invasive breast carcinoma. Breast Cancer Res Treat. 2018; 172 (3): 671-677.
24. Sparano JA, Gray RJ, Ravdin PM, Makower DF, Pritchard KI, Albain KS et al. Clinical and genomic risk to guide the use of adjuvant therapy for breast cancer. N Engl J Med. 2019; 380: 2395-2405.

25. Zhang Y, Zhou Y, Mao F,Yao R, Sun Q. Ki67 index, progesterone receptor expression, histologic grade and tumor size in predicting breast carcinoma recurrence risk: a consecutive cohort study. Cance Comm (Lond). 2020; 40 (4): 181-193.

26. Henderson J, Adams P, Barber K. Factors determining anthracycline use in hormone receptor positive, early-stage breast cancer. Clin Breast Cancer. 2019; 19: e475-e480.

27. Jasem J, Fisher C, Amini A, Shagisultanova E, Ravinotvich R, Borges $\mathrm{V}$ et al. The 21-gene recurrence score assay for nodepositive, early-stage breast cancer and impact of RxPONDER trialon chemotherapy decision-making: have clinicians already decided? J Natl Compr Canc Netw. 2017; 15 (4): 494503.

28. Albain KS, Paik S, Van't Verr L. Prediction of adjuvant chemotherapy benefit in endocrine responsive, early breast cancer using multigene assays. Breast. 2009; (Suppl 3); S141-S145. 